\title{
CORRESPONDENCE
}

\section{Treatment strategies for Cheyne-Stokes respiration}

\section{To the Editors:}

In a recent issue of the European Respiratory Journal, GABOR et al. [1] reported the effects of standard cardiac resynchronisation therapy (CRT) on nocturnal central sleep apnoea (CheyneStokes respiration). CRT was shown to reduce Cheyne-Stokes respiration significantly in six out of 10 patients.

The study was performed in Canada between 1999 and 2002, which is the same era as when new generations of betablockers (e.g. metoprolol, carvedilol) were introduced into the market. The authors declare that the medication remained unchanged during the study, but that might be true for substance classes only and not for the type of beta-blocker. Substitution of beta-blockers had been a routine measure at that time in many industrialised countries [2].

According to our own data (currently unpublished), there seems to be a statistically significant and clinically relevant effect of high-dose beta-blocker treatment on the prevalence and severity of nocturnal central apnoeas and hypopnoeas. It could be demonstrated in three well-matched cohorts (no betablocker $n=16$; metoprolol $n=16$; carvedilol $n=13$ ) of chronic heart failure patients (New York Heart Association II and III) that both beta-blockers, i.e. metoprolol and carvedilol, could significantly reduce the combined apnoea-hypopnoea index $(19.5 \pm 14.4$ versus $7.4 \pm 8.5$ versus $8.7 \pm 8.1$ per hour of sleep, respectively).

We suggest that the results of the study by GABOR et al. [1] should be related to the presence, dosage and type of betablocking medication in order to discriminate between the effects of cardiac resynchronisation therapy and potential pharmacological influences.

\section{T. Köhnlein and T. Welte}

Pulmonary and Intensive Care Medicine, Hannover Medical School, Hannover, Germany.

\section{REFERENCES}

1 Gabor JY, Newman DA, Barnard-Roberts V, et al. Improvement in Cheyne-Stokes respiration following cardiac resynchronisation therapy. Eur Respir J 2005; 26: 95-100.

2 Franciosa JA, Massie BM, Lukas MA, et al. Beta-blocker therapy for heart failure outside the clinical trial setting: findings of a community-based registry. Am Heart J 2004; 148: 718-726.

DOI: 10.1183/09031936.06.00084405

\section{Endoscopic ultrasound-guided biopsy in the chest}

\section{To the Editors:}

We read with interest the editorial comment on endoscopic ultrasound-guided fine-needle aspiration (EUS-FNA) biopsy in the chest by VILMANN and LARSEN [1].

With conviction, they claim the importance of EUS-FNA in the work-up of enlarged mediastinal lymph nodes, especially in lung cancer patients. They even state that: "we really do not need additional proof before EUS-FNA is considered the gold standard for invasive staging of nonsmall cell lung cancer and for diagnosis of posterior mediastinal lesions".

However, as they also state in their discussion, no studies have actually compared mediastinoscopy (the current gold standard) and EUS-FNA in a controlled and blinded study design. Moreover, other concerns about EUS-FNA can be raised, which are not mentioned in their comments, such as the following.

First, according to VILMANN and LARSEN [1], the sensitivity of EUS-FNA biopsies of mediastinal lymph nodes is very high. This may be true for the nodes within reach of the transoesophageal needle. Due to the anatomical position of the oesophagus, only nodes in the distal and posterior mediastinum can be reached. The right-sided mediastinal nodes $2 \mathrm{R}$ and $4 \mathrm{R}$ cannot be reached by EUS-FNA in a significant number of cases, as is confirmed in the article by RINTOUL et al. [2], published in the same issue of the European Respiratory Journal. Here the diagnosis of the $2 \mathrm{R}$ and $4 \mathrm{R}$ nodes was obtained using a transbronchial approach [2].

Before EUS-FNA is claimed to be the gold standard for invasive staging of nonsmall cell lung cancer, a prospective study should demonstrate the number of cases of enlarged lymph nodes which are out of reach for EUS-FNA, but can be reached by mediastinoscopy, the current gold standard. Such a study would demonstrate a more accurate sensitivity of EUSFNA in an unselected group of lung cancer patients with enlarged lymph nodes.

Secondly, VILMANN and LARSEN [1] begin their editorial with the statement that "tissue diagnosis of pathological lesions located in the mediastinum has been difficult to obtain by nonsurgical methods". However, they forget to mention transbronchial needle aspiration (TBNA), a technique known since 1949 [3], which has been used with the flexible bronchoscope since 1979 [4]. This nonultrasound-guided procedure has a diagnostic sensitivity of $>70 \%$ in many studies [5-7]. 
Moreover, these results are not reserved for expert centres alone. After 24 months of training, the diagnostic sensitivity of pulmonologists without experience in TBNA rose from 24 to $78 \%$ [5].

TBNA has some important advantages. 1) It can be performed during the bronchoscopic procedure that is already necessary in the work-up of patients suspected for lung cancer. The extra time is limited to a few minutes and the additional costs of a TBNA needle are $\sim 90 €$. 2) The use of ultrasound is a necessity in the oesophagoscope as there are no anatomical landmarks in the oesophagus. During bronchoscopy, one can rely on several endobronchial landmarks to localise lymph nodes. In the only randomised trial in the literature concerning the use of ultrasound-guided versus "blind" TBNA, there was no significant difference in the diagnostic yield of subcarinal lymph nodes [8]. 3) It is a cost-effective procedure which may diagnose N3 disease in an important number of cases, and consequently avoid mediastinoscopy $(1,550 €$ in our hospital) [6]. 4) From the patient's point of view, if TBNA performed during the necessary bronchoscopy is diagnostic, no additional endoscopic procedures are needed. After all, who would volunteer for oesophagoscopy?

In an editorial comment on the endoscopic approach of mediastinal lymph nodes, the vast literature on the excellent results of TBNA should not be neglected.

Before endoscopic ultrasound-guided fine-needle aspiration is proclaimed as the new gold standard, and pulmonologists are advised to learn oesophagoscopy, we should first learn to perform a routine transbronchial needle aspiration during the bronchoscopy. This approach is cost-effective and limits the number of endoscopic procedures, which may even be unnecessary. Your patient will be grateful to you!

\section{J. Janssen*, S. Gasparini" ${ }^{\#}$ and A. Mehta}

*Canisius Wilhelmina Ziekenhuis, Nijmegen, the Netherlands.

\#Azienda Ospedaliero-Universitaria, Ferrara, Italy. "The Cleveland Clinic Foundation, Cleveland, $\mathrm{OH}$, USA.

\section{REFERENCES}

1 Vilmann P, Larsen SS. Endoscopic ultrasound-guided biopsy in the chest: little to lose, much to gain. Eur Respir J 2005; 25: 400-401.

2 Rintoul RC, Skwarski KM, Murchison JT, Wallace WA, Walker WS, Penman ID. Endobronchial and endoscopic ultrasound-guided real-time fine-needle aspiration for mediastinal staging. Eur Respir J 2005; 25: 416-421.

3 Schiepatti E. La puncion mediastinal a traves del espolon traqueal [Trans-tracheal aspiration of the mediastinum]. Rev As Med Argent 1949; 663: 497-499.

4 Oho K, Kato H, Ogawa I, Hayashi N, Hayata Y. A new needle for transfiberoptic bronchoscope use. Chest 1979; 76: 492.

5 Rodriguez de Castro F, Diaz Lopez F, Serda GJ, Lopez AR, Gilart JF, Cabrera Navarro P. Relevance of training in transbronchial fine-needle aspiration technique. Chest 1997; 111: 103-105.
6 Hermens FHW, Van Engelenburg TCA, Visser FJ, Thunnissen FBJM, Termeer R, Janssen JP. Diagnostic yield of transbronchial histology needle aspiration in patients with mediastinal lymph node enlargement. Respiration 2003; 70: 631-635.

7 Schenk DA, Chambers SI, Derdak S, et al. Comparison of the Wang 19 gauge and 2 gauge needles in the mediastinal staging of lung cancer. Am Rev Respir Dis 1993; 147: 1251-1258.

8 Herth F, Becker HD, Ernst A. Conventional vs endobronchial ultrasound-guided transbronchial needle aspiration: a randomized trial. Chest 2004; 125: 322-325.

DOI: 10.1183/09031936.06.00069605

\section{From the authors:}

We read with interest the letter to the Editors by J. Janssen and coworkers regarding our editorial comment on endoscopic ultrasound-guided fine-needle aspiration biopsy (EUS-FNA), which was featured in the March issue of the European Respiratory Journal [1].

Our comment resumes the present status concerning EUS-FNA in the chest [1]. All available studies indicate that EUS-FNA is promising in lung cancer (LC) staging. However, as stated several times in our comment, final conclusions have to await large blinded, randomised comparative studies. So, what do we do in the mean time when dealing with the major cancerrelated cause of death with a terrible prognosis, which is almost unchanged during the past 40 yrs?

Concerning the right paratracheal regions $(2 R+4 R)$, we agree that these regions are not as easily accessible by EUS-FNA when compared with the left-sided mediastinal regions. At present, mediastinoscopy (MS) may be the best method for these regions. According to our experience, an important number of patients with $2-4 \mathrm{R}$ disease can be diagnosed with advanced inoperable disease by EUS-FNA [2].

With regards to transbronchial needle aspiration (TBNA) biopsy, we do agree that the results of this minimally invasive method should not be neglected and TBNA should be performed during initial bronchoscopy in patients suspected of LC. Only patients considered as surgical candidates after TBNA should undergo further invasive staging. That is the current practice at our centre and, we suppose, most other centres. However, in spite of TBNA and MS, $~ 40 \%$ of intended curative operations for LC are either explorative without resection or followed by recurrence. This is due to undetected advanced disease prior to surgery. Therefore, new and minimally invasive methods are needed to improve LC staging.

In this context, we found EUS-FNA promising. As EUS-FNA is less invasive, has fewer complications and does not require general anaesthesia, we suggest that EUS-FNA is performed as 